\title{
IDH1 Protein Variant
}

National Cancer Institute

\section{Source}

National Cancer Institute. IDH1 Protein Variant. NCI Thesaurus. Code C117278.

A variation in the amino acid sequence for the isocitrate dehydrogenase [NADP] cytoplasmic protein. 